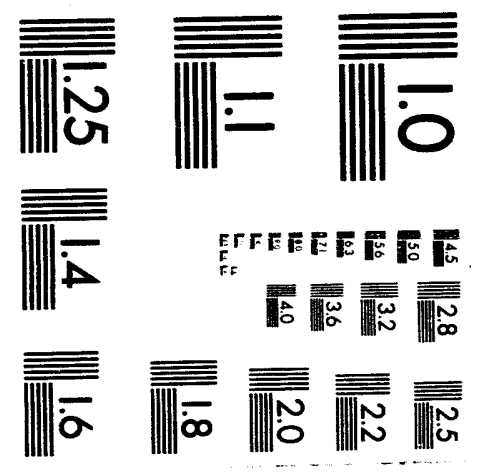



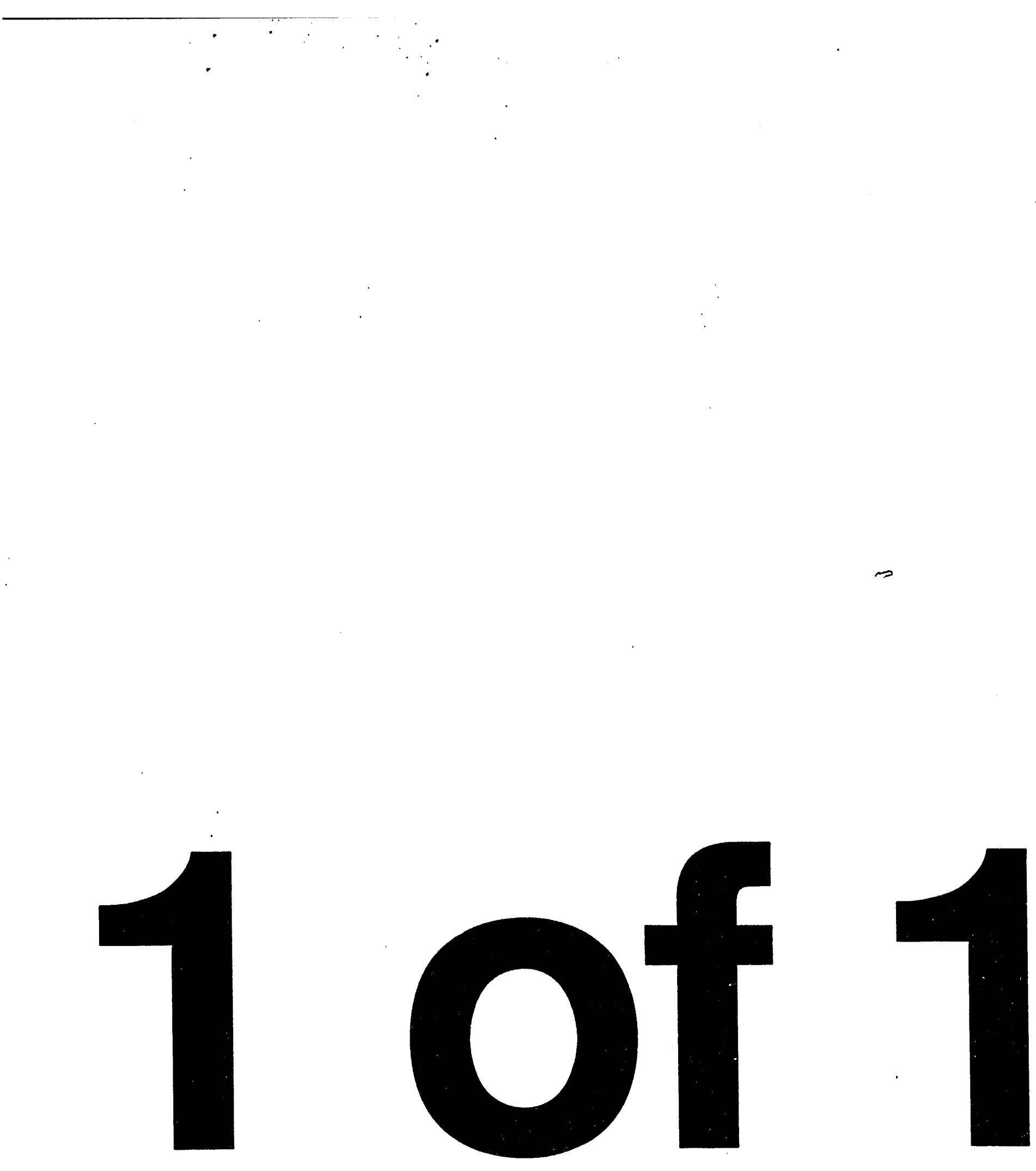
LA-UR- $93-4474$

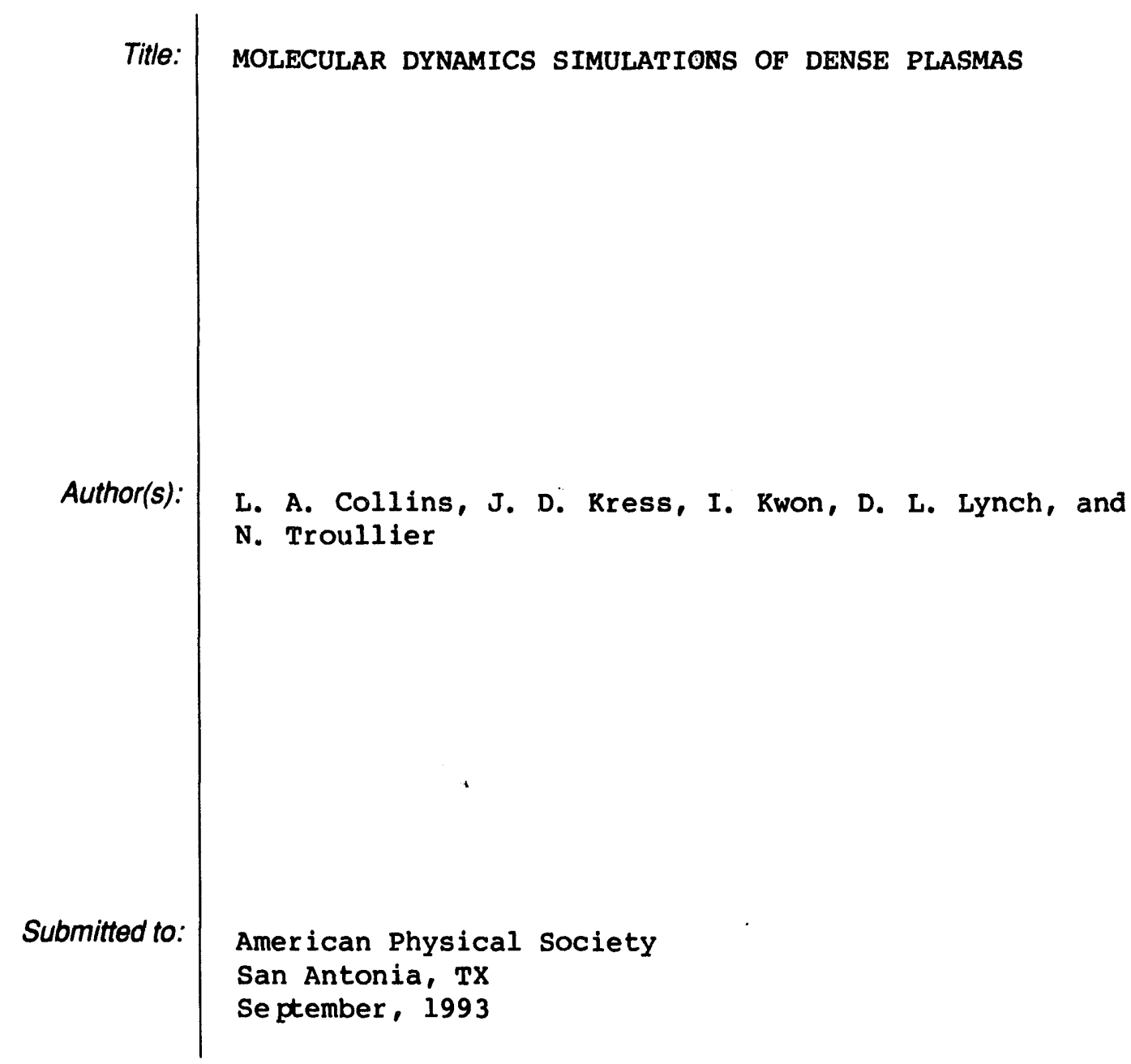

\section{DISCLAIMER}

This report was prepared as an account of work sponsored by an agency of the United States Government. Neither the United States Government nor any agency thereof, nor any of their employees, makes any warranty, express or implied, or assumes any legal liability or responsibility for the accuracy, completeness, or usefulness of any information, apparatus, product, or process disclosed, or represents that its use would not infringe privately owned rights. Reference herein to any specific commercial product, process, or service by trade namc, trademark, manufacturer, or otherwise does not necessarily constitute or imply its endorsement, recommendation, or favoring by the United States Government or any agency thereof. The views and opinions of authors expressed herein do not necessarily state or reflect those of the United States Government or any agency thereof.

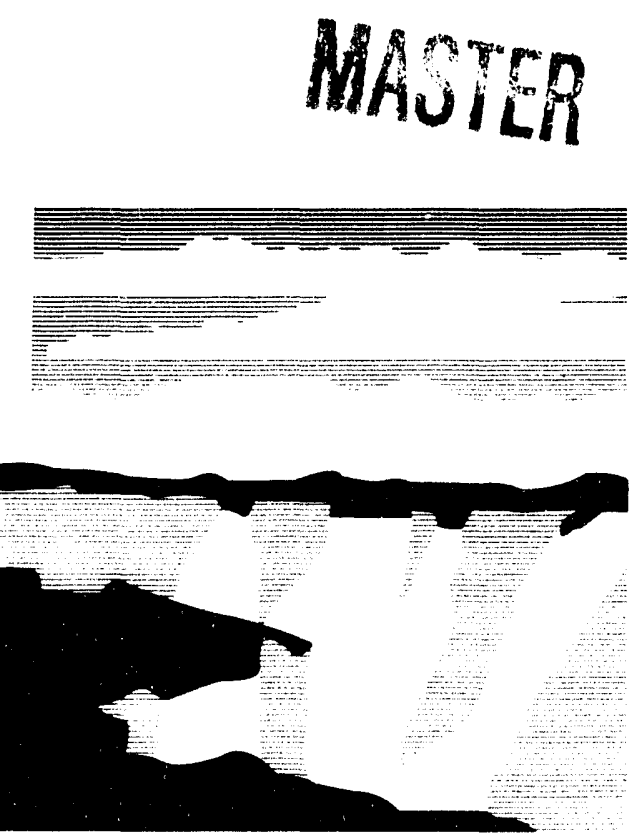

\section{Los Alamos} NATIONAL LABORATORY

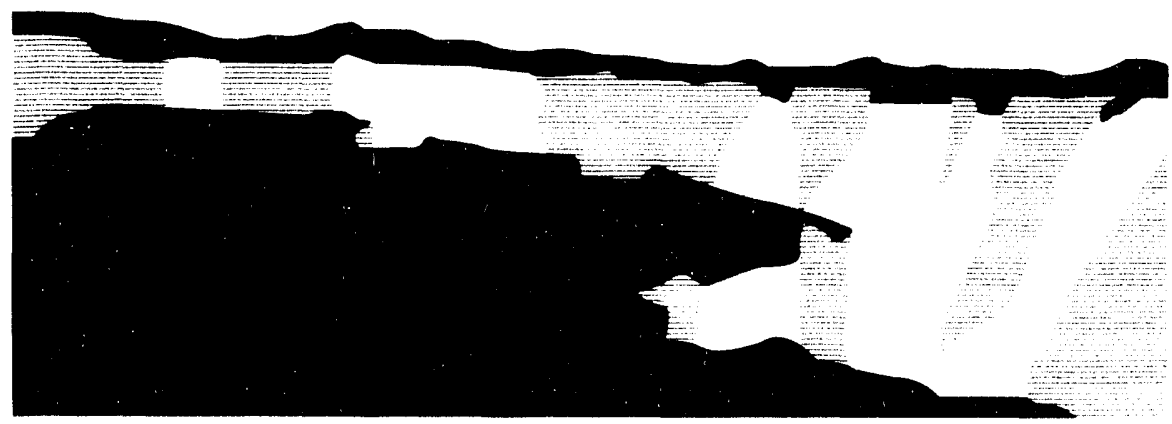

Los Alamos National Laboratory, an aftirmative action/equal opportunity empióyer, is operated by the University of California for the U.S. Department of Energy under contract W-7405-ENG-36. By acceptance of this article, the publisher recognizes that the U.S. Government retains a nonexclusive, royalty-free license to publish or reproduce the published form of this contribution, or to allow others to do so, for U.S. Government purposes. The Los Alamos National Laboratory requests that the publisher identify this article as work performed under the auspices of the U.S. Department of Energy. 


\title{
MOLECULAR DYNAMICS SIMULATIONS OF DENSE PLASMAS
}

\author{
L. Collins, J. Kress, and I. Kwon \\ Los Alamos National Laboratory, Los Alamos, NM 87545 \\ D. Lynch \\ Thinking Machines Corp., Cambridge, MA 02142
}

N. Troullier

Department of Computer Science and Dept. of Chemical Engineering

and Material Science, University of Minnesota, Minneapolis, MN 55455

\begin{abstract}
We have performed quantum molecular dynamics simulations of hot, dense plasmas of hydrogen over a range oi temperatures $(0.1-5 \mathrm{eV})$ and densities $(0.0625-$ $5 \mathrm{~g} / \mathrm{cc}$ ). We determine the forces quantum mechanically from density functional, extended Huckel, and tight binding techniques and move the nuclei according to the classical equations of motion. We determine pair-correlation functions, diffusion coefficients, and electrical conductivities. We find that many-body effects predominate in this regime. We begin to obtain agreement with the OCP and Thomas-Fermi models only at the higher temperatures and densities.
\end{abstract}

\section{INTRODUCTION}

Dense plasma has an ambiguous meaning, covering a vast range of temperatures and densities. Both high-temperature Tokamak $\left(10^{14}\right.$ particles/cc) and Inertial Confinement Fusion $\left(10^{25} / \mathrm{cc}\right)$ plasmas fall under this rubric as do some cool processing media. We focus our attention on very dense plasmas ${ }^{1,2}$ near or above solid densities. For such regimes, the collective quantum mechanical nature of the whole system becomes paramount. At low densities $\left(10^{15}-10^{18} / \mathrm{cc}\right)$, we may accurately model the medium through the solution of kinematic equations based on isolated, independent atomic events. No direct effects of the plasma as a whole appear in the particle and photon interactions. $A$ s the density increases $\left(10^{19}\right.$. $10^{22} / \mathrm{cc}$ ), the surrounding media begins to exert an influence on the basic atomic processes. These effects are generally included through perturbative techniques that adjust the isolated atomic interactions for the effects of nearby ions and electrons. The plasma microfields and continuum lowering fall in this category of perturbed-atom models. However, compressing the plasma further $\left(10^{23}-10^{25} / \mathrm{cc}\right)$, leads to an entirely new regime in which the collective effects of all atoms in the media must be accurately represented. In addition, since the wavefunctions of the various atoms strongly overlap, a quantum mechanical(QM) treatment becomes necessary. The "plasma" resembles a QM fluid at elevated temperatures in which all atoms must be treated democratically.

The earliest demonstrations of the importance of collective media effects in accurately modeling dense plasmas arose from quantum chemistry calculations ${ }^{3,4}$ on large, static collections of atoms. These calculations indicated that certain 
properties, such as electronic band widths, required sample sizes of hundreds of atoms to properly reach convergence. Since the forces on the individual ions also depend upon these same quantum mechanical electronic properties, the evidence strongly supported a similar sample-size effect upon the plasma dynamics. Time-dependent Hartree-Fock methods remain the most effective means of treating electrons and ions on an equal basis. However, since their implementation lies well outside current computational and budgetary constraints, all current treatments rely on the Born-Oppenheimer(BO) approximation. In this case, the nuclear and electronic motions are decoupled. We temporally advance the system by a two-step procedure: 1) determination of the forces on ions from a fully quantum mechanical(QM) electronic structure calculation and 2) movement of the ions by classical molecular dynamics methods based on the QM forces. We therefore can evolve the system in time and determine such properties as diffusion coefficients, electrical conductivities, pair correlation functions, and the density-of states from which radiative properties can be derived. This quantum molecular dynamics(QMD) scheme allows an intricate investigation of the basic properties of dense plasma systems.

Many techniques have been developed to implement this QMD treatment of hot,dense media. Electronic structure methods range over a wide variety of techniques from the elaborate Hartree-Fock ${ }^{5}$ and density functional ${ }^{6-8}$ to the semiempirical tight-binding, ${ }^{9}$ extended Huckel, ${ }^{7}$ and Thomas-Fermi. ${ }^{10}$ Most determine forces directly from a diagonalization of the electronic Hamiltonian at each time step although some, such as the Car-Parrinello ${ }^{11}$ techniques, apply a modified form of simulated annealing over a succession of temporal points. Whatever the procedure, all current methods operate on or near the $\mathrm{BO}$ surface. This restriction can be modified to include temperature effects by invoking local thermodynamical equilibrium. The energy levels are occupied according to a Fermi-Dirac distribution based on the ionic kinetic temperature; the $\mathrm{QM}$ forces on the ions are then determined from this enhanced distribution. With these procedures, we can study the dynamical nature of a hot, dense plasma.

\section{FORMALISM}

Since we have discussed our procedures and methods in greater detail elsewhere, ${ }^{7,12}$ we give only a brief outline of the basic techniques. We choose as our standard model a collection of $\mathrm{N}_{a}$ ions and $\mathrm{N}_{e}$ electrons confined in a cubic reference cell of length $\mathrm{L}$. We advance the system in time by a two-step prescription: 1) determine the forces on the ions from a quantum mechanical $\mathrm{N}_{\mathrm{e}}$-electron structure calculation on the fixed nuclear configuration, and 2) move the ions according to the classical equations-of-motion based on the QM forces. Repeating this procedure allows a temporal evolution of the reference system. With the general prescription in hand, we turn to the specific techniques used in executing these two steps.

We begin the simulation with a configuration of high symmetry such as $\mathrm{BCC}$ or FCC and impart to each atom a random velocity constrained to a MaxwellBoltzmann distribution at a set temperature $\mathrm{T}_{i}$ by a Box-Muller prescription. The 
classical equations of motion are integrated by a velocity Verlet propagator, ${ }^{13,14}$ which is third order in time and second order in velocity. This procedure advances the positions and velocities of the particles a small amount for each time step. We maintain a constant density within the reference cell and therefore replace any particle that exits the cell with a corresponding one entering. However, we do invoke two different temperature conventions. For the microcanonical ensemble, we allow the temperature to vary freely. The degree to which the total energy remains constant provides a measure of the accuracy of the temporal propagation. On the other hand, for a canonical ensemble, we fix the temperature at a prescribed value by a simple velocity scaling technique. At designated time steps, we scale the particle velocities by the square root of the ratio of the average kinetic to prescribed temperature. The collection of particle positions and velocities at specific time steps forms the trajectory of the system. For statistical accuracy, many trajectories should be employed and suitable averages made over the whole collection. From the trajectories, we determine the mean-square displacement(MSD) and velocity(VAC) autocorrelation functions, which relate to the self-diffusion coefficients, and the pair-correlation functions, which characterize the structure of the media. ${ }^{13,14}$

We calculate the quantum mechanical forces on the ions by several techniques of varying sophistication: the density functional(DF),${ }^{15-16}$ extended Huckel(EH), ${ }^{17}$ and tight binding(TB). ${ }^{18}$ In each case, we perform calculations for $\mathrm{N}_{e}$ electrons on a fixed configuration of $\mathrm{N}_{a}$ ions within the reference cell. The DF forms the most accurate approach, representing direct, exchange, and correlation effects. By the same token, such accuracy comes at a high computational price, allowing only small systems to be treated for the long trajectories needed to produce meaningful dynamical properties. On the other hand, the semi-empirical approaches, EH and TB, sacrifice detailed representations of the interactions for computational efficiency. Therefore, we can investigate far larger samples of atoms with these methods, giving more precise structural and macroscopic properties. By employing the DF as a benchmark of the semi-empirical models, we can combine accuracy with speed to give a thorough representation of the plasma environment.

In all three cases, we form and diagonalize an effective hamiltonian matrix to determine eigenvalues and eigenvectors. The force on a given atom depends on the derivative with respect to the nuclear coordinate of the total energy of the system, which, in turn, depends on the occupied molecular orbitals. At low temperatures, filling the lowest eigenstates each with two electrons to account for spin until all $\mathrm{N}_{e}$ have been depleted gives the appropriate occupancy. However, to introduce finite temperature effects, we assume local thermodynamical equilibrium(LTE) in which the ionic kinetic temperature $\left(\mathrm{T}_{i}\right)$ equals that of the electrons $\left(\mathrm{T}_{e}\right)$. We then populate the eigenstates according to a Fermi-Dirac distribution at $T_{i}$. In this manner, the effects of excited and continuum states are included implicitly into the calculation of the force and similarly into the dynamics of the system.

We invoke the local density approximation(LDA ${ }^{16}$ t,o the DF formulation and expand in terms of a plane wave(reciprocal lattice vectors) basis. We include all reciprocal lattice vectors up to $\mathrm{g}_{\max }$, which sets the energy cut-off in Rydbergs 
at $\mathrm{E}_{\max }=\mathrm{g}_{\max }^{2} / 2$, and integrate over $\mathrm{k}$-space only at the gamma point. Since we employ a large real-space reference cell, this single-point $k$-space integration is sufficiently accurate over the corresponding small Brillouin zone. We generally treat the valence electrons explicitly within the LDA and represent the effects of the core electrons by a pseudopotential. Construction of the pseudopotential employs the Troullier-Martins ${ }^{19}$ scheme transformed by the Kleinman-Bylander procedure from semi-local to nonlocal form. Being a self-consistent prescription in which the hamiltonian matrix elements depend implicitly upon the charge density(eigenvectors), the LDA must be iterated to convergence at each time step. A more thorough description of these procedures can be found in several review articles. ${ }^{15-16}$

The extended Huckel and tight-binding procedures have much in common. In both, we replace the complex hamiltonian with one based on simple semi-empirical models representing the valence electrons. A model pair-potential incorporates the effects of the core electrons and the nuclear repulsions. In the $\mathrm{EH},{ }^{17}$ we expand the $\mathrm{N}_{e}$-electron wavefunction in a simple product of molecular spin orbitals. These molecular orbitals are in turn expanded in a basis of contracted gaussian functions(GTO) centered on the nuclei. We then approximate the resulting hamiltonian matrix elements, which generally contain multi-electron and multi-center integrals, by a particularly simple form:

$$
\begin{gathered}
H_{i i}=\epsilon_{i} \\
H_{i j}=0.5 K\left(\epsilon_{i}+\epsilon_{j}\right) S_{i j},
\end{gathered}
$$

where $\epsilon_{i}$ represents the valence orbital energy, $S_{i j}$, the overlap integral between basis functions $i$ and $j$, and $K$ a scaling parameter. Applying a variational method to the minimization of the total electronic energy leads to a generalized eigenvalue form:

$$
\mathbf{H c}=\mathbf{S c} \lambda
$$

whose solutions $\mathrm{c}$ are the expansion coefficients of the GTO basis. By adjusting the valence energies $\epsilon, \mathrm{K}$, and the GTO exponential coefficients to give agreement with certain molecular and solid properties, we can obtain a reasonable representation of the composite $\mathrm{N}_{a}$-atom reference system. For extended media as fluids and plasmas, we must augment the usual EH formulation. First, we model the bulk properties by employing a Block-wave form ${ }^{20}$ for the $\mathrm{EH}$ wavefunction. This procedure involves a converged sum over all contributing image cells. For systems under high compression, this sum may extend over several tiers of such cells, incorporating thousands of atoms. Second, we extend the usual minimum basis set to include more flexible types of functions. Such flexibility becomes essential in modeling very dense, hot environments. Therefore, for hydrogen, we use both $1 \mathrm{~s}$ and $\mathrm{s}^{\prime}$ functions and for an alkali, a (ns,np) basis. Since the hamiltonian has no explicit dependence of the eigenvectors(charge density), the $\mathrm{EH}$ procedure eliminates the need for a self-consistency procedure. Therefore, we need only perform one matrix diagonalization for each time step. This fact, coupled to 
the simplicity and speed of evaluating the matrix elements, makes the $\mathrm{EH}$ an extremely efficient and computationally expedient scheme.

In the TB method, ${ }^{9}$ the hamiltonian matrix elements have the following form:

$$
H_{\alpha, \beta}=V_{\alpha, \beta}\left(r_{\alpha, \beta}\right),
$$

where the composite label $\alpha$ designates both a particular atomic center and an orbital symmetry, and $r_{\alpha, \beta}$ gives the distance between the two sites. We assume a form for $\mathrm{V}$ in terms of several free parameters and adjust these parameters to give the best fits to molecular and solid data. For hydrogen, we base out initial construction upon the diatomic molecule $\mathrm{H}_{2}$ since the TB matrix elements can be straightforwardly identified with the diatomic ground and excited state potential energy curves. We have designed two model potentials. The first contains only 1s character on each atom and closely resembles one derived by Skinner and Pettifor $^{21}$ from Harris-Foulkes DF theory. $\mathrm{We}^{7}$ have augmented this simple form with an $\mathrm{s}^{\prime}$ component, fit to the E, $\mathrm{F}$ excited state of $\mathrm{H}_{2}$ with proper symmetry. This enhanced TB potential gives a more extended and diverse eigenspectrum, essential to modeling high temperatures. The ss' TB form is in excellent agreement with the $\mathrm{H}_{2}$ ground state curve and predicts the proper energetic ordering among the crystalline symmetries of the hydrogenic solid. Like the EH prescription, the TB approach is noniterative and computationally efficient. The details of both prescriptions together with the defining parameters are found elsewhere ${ }^{7,12}$.

In addition to the pair- and auto- correlation functions, the electrical conductivity gives important information about the conditions within the plasma. The dc conductivity follows from the Kubo-Greenwood formula ${ }^{22}$ as

$$
\sigma_{E}=\frac{2 \pi^{2} e^{2} \hbar^{3} \Omega}{m^{2}} \int \frac{\partial f(E)}{\partial E}|D|_{a v}^{2}[N(E)]^{2} d E
$$

where $\Omega$ is the atomic volume, $|D|_{a v}^{2}$ is the square expectation value of the electron velocity operator $\frac{\partial}{\partial x}, f(E)$ is the Fermi-Dirac distribution, and $N(E)$ is the electronic density of states per unit volume. An explicit value for the conductivity can then be evaluated from a temporal average over the MD trajectory. Since ill three QM structure methods produce a discrete spectrum, we broaden each eigenvalue by a gaussian function to determine the continuous density-of-states required in Eq.(5). We usually select the width of the gaussian, F, proportional to the kinetic temperature $\mathrm{k}_{B} \mathrm{~T}_{i}$. At low temperatures, we can further simplify ${ }^{22}$ Eq.(5). The function $\frac{\partial f}{\partial E}$ resembles a delta function of a width $\mathrm{k}_{B} \mathrm{~T}_{i}$ about the Fermi energy $\epsilon_{F}$ and therefore extracts values of the integrand only very near $\epsilon_{F}$. In general, we can determine the velocity-dipole matrix elements from the eigenfunctions of the LDA, TB, or EH calculations at a particular time step. We use the random phase approximation to estimate the value of the velocity-dipole element as $a / \Omega$ with a the average neighbor distance. At $T_{i}=0$, we determine the Fermi energy as the highest occupied level formed by filling the lowest eigenstates with all available $\left(\mathrm{N}_{e}\right)$ pairs of electrons. For a small, finite $\mathrm{T}, \epsilon_{F}$ must be determined self-consistently and moves above the $\mathrm{T}=0$ position. Therefore, the conductivity depends critically on those states very near and particularly just above the Fermi 
energy. We readily observe that these approximations lead to a close link between the conductivity and the band gap. We expect appreciable conductivity when the band gap and the kinetic energy are comparable.

\section{RESULTS AND DISCUSSION}

While our main objective rests with modeling hot, dense hydrogen plasmas, very little experimental or theoretical results exist with which to compare. However, the alkali systems, which have one diffuse electron outside of a tightly closed core, do resemble hydrogen in many essential aspects, especially in regimes in which the core plays no predominant role. Both theoretical and experimental results abound for the alkali systems. Therefore, as a first test our QMD schemes, we have performed $\mathrm{DF}$ and $\mathrm{EH}$ calculations on alkali $(\mathrm{Li}, \mathrm{Na})$ liquids at temperatures of a few hundred degrees $\mathrm{K}$ above melt. For lithium, ${ }^{7,12}$ we obtained excellent agreement $(10 \%)$ in the pair-correlation function and the self-diffusion coefficient with other LDA calculations ${ }^{23}$ and with experiments. ${ }^{24}$ The EH also produces dynamical quantities within this same percentage range. For sodium, ${ }^{12}$ the agreement between density functional methods ${ }^{25}$ remains excellent although that with experiment ${ }^{24}$ is somewhat poorer(15\%). The EH gives diffusion coefficients within about $20 \%$ of the DF. These results tend to confirm the validity of both the EH and DF techniques.

We also obtained very good agreement with recent LDA calculations for hydrogen plasmas at low temperatures. Our results ${ }^{7}$ for 54 atoms compare favorably with those of $\mathrm{Hohl}$ et. al., ${ }^{8}$ who use 64 atoms. At a fixed temperature $(0.1 \mathrm{eV}$, $1160 \mathrm{~K})$, we obtain a molecular $\mathrm{H}_{2}$ fluid for low densities $(\rho \leq 0.75 \mathrm{~g} / \mathrm{cc})$. Between 0.75 and $1.25 \mathrm{~g} / \mathrm{cc}$, we observe pressure dissociation of $\mathrm{H}_{2}$, characterized by the gradual disappearance of the distinctive molecular peak in the pair distribution function at $1.4 \mathrm{bohr}$. At higher densities, we find a disordered atomic system. Similarly, by fixing the density (e.g., $\rho=0.25 \mathrm{~g} / \mathrm{cc}$ ) and raising the temperature $(\mathrm{T}=0.35 \mathrm{eV})$, we also obtain dissociation but by kinematic means. Our ss' TB model $^{7}$ gives similar results leading to a slightly higher density for pressure dissociation. These observations generally agree with limited experimental results in this high density, low temperature regime.

In this low temperature range, we have also calculated the dc conductivity as a function of density. We have employed the approximate formula due to Mott at a temperature of $0.1 \mathrm{eV}$ for both $1 \mathrm{~s}$ (Skinner-Pettifor) and ss' $\mathrm{TB}$ models. We employed MD runs of 250 atoms and determined the eigenvalue spectrum as an average over every tenth time step. We broadened the discrete spectrum by $\mathrm{F}=0.1 \mathrm{eV}$ and performed the integration in Eq.(5) by a trapezoidal quadrature. We present the results of the dc conductivity in Fig.1. We observe a transition from insulator to conductor at about the same density regime of pressure dissociation. At this point, the band gap becomes equivalent to or less than the thermal broadening of the discrete states, leading to the indicated transition. The conductivity reaches a reasonably high value; for comparison, copper has a $\sigma_{E}$ of $6 \times 10^{5}(\Omega-\mathrm{cm})^{-1}$. While based on approximate models, these calculations of conductivity do provide a gencral appraisal of the behavior in regions of high compression. 


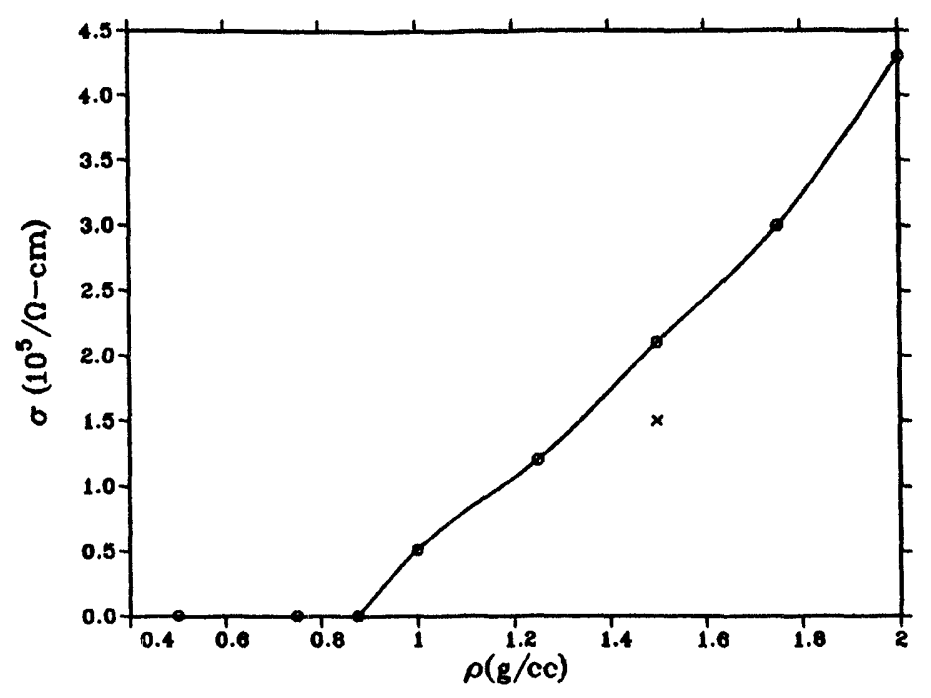

Figure 1: dc conductivity as a function of density for TB models: line(1s), $\operatorname{cross}\left(\mathrm{ss}^{\prime}\right)$.

We examine the higher temperature regime with both the DF-LDA and TB methods. We have particular interest in comparing properties with simpler models such as the one-component plasma $(\mathrm{OCP})^{2}$ and the Thomas-Fermi(TF). ${ }^{10}$ The OCP should represent the conditions within the plasma at high temperatures and densities since the model consists of point ions within a uniform electron background. We generally characterize many of the properties of the OCP by a single parameter $\Gamma$, the ratio of the Coulomb to kinetic energies:

$$
\Gamma=Z^{2} /\left(r_{s} k_{B} T_{i}\right)
$$

where $\mathrm{Z}$ is the bare ionic charge, $\mathrm{r}_{s}$ is the ion sphere radius, and $\mathrm{T}_{i}$ is the ion temperature. A $\Gamma$ value of unity usually divides plasmas between weakly and strongly coupled. For all our applications, we reside in the strongly-coupled regime. The Thomas-Fermi provides a better model of the electron-ion interaction compared to the OCP but one still deficient in many respects due to its lack of binding. In Fig. 2, we compare our DF and ss' TB self-diffusion coeflicients for three temperatures as a function of ion density. For the density spanned $(0.25 \leq \rho \leq 3.00 \mathrm{~g} / \mathrm{cc})$, the $\Gamma$ parameter has a range $10-30$ for $T=1 \mathrm{eV}, 4-9$ for $3 \mathrm{eV}$, and $2.5-5.5$ for $5 \mathrm{eV}$. At the lowest temperature, we find very good agreement between the LDA and TB results above about $1 \mathrm{~g} / \mathrm{cc}$. The differences at lower densities probably arise from the short-range nature of the TB potential. At these densities, the atoms reside farther apart and the intermediate component of the potential becomes more important. In this distance range, the TB potential becomes less attractive than the DF, not having as accurate a representation of the longer-range Coulomb components. We note that the OCP performs very poorly in this regime since the many-body interactions remain important. As the temperature rises, the agreement between the TB and OCP improves. In this case, the elevated temperature places more distribution in the excited and continuum states. Since these states have a much larger overlap due to their diffuse nature, the media begins to resemble an OCP. A similar condition arises as the density becomes larger. We 
should caution about attributing too much import to $\Gamma$, even at the higher temperature, since screening and many-body effects still play an important role. We have also compared with TF models. Again at the low temperature, high density range, the agreement is poor, but improves as the temperature increases. While we have reported results for $\mathrm{H}$, we can obtain values for the various thermodynamical properties for fluids of its isotopes by simple scaling rules. This result arises from the electronic potential being independent of isotopic mass and the equations-of-motion being derived in terms of a scaled mass. The relationships are as follows:

- $\mathrm{T}_{d}=\mathrm{T}_{t}=\mathrm{T}_{H}$

- $\rho_{d}=2 \rho_{H}$ and $\rho_{t}=3 \rho_{H}$;

- $\mathrm{D}_{d}=\mathrm{D}_{H} / \sqrt{2}$ and $\mathrm{D}_{t}=\mathrm{D}_{H} / \sqrt{3}$.

Therefore, Fig.2 equally applies for $\mathrm{D}$ and $\mathrm{T}$ plasmas with the axes appropriately scaled.

In conclusion, we have developed a quantum molecular dynamics approach to simulating hot, dense plasmas. We obtain results for a density $(0.0625-5 \mathrm{~g} / \mathrm{cc})$ and temperature $(0.1-5 \mathrm{eV})$ in which many-body effects play an important role, indicating that models beyond OCP and Thomas-Fermi must be employed.

Work performed under the auspices of the U.S. Department of Energy through the Theoretical Division of the Los Alamos National Laboratory. 

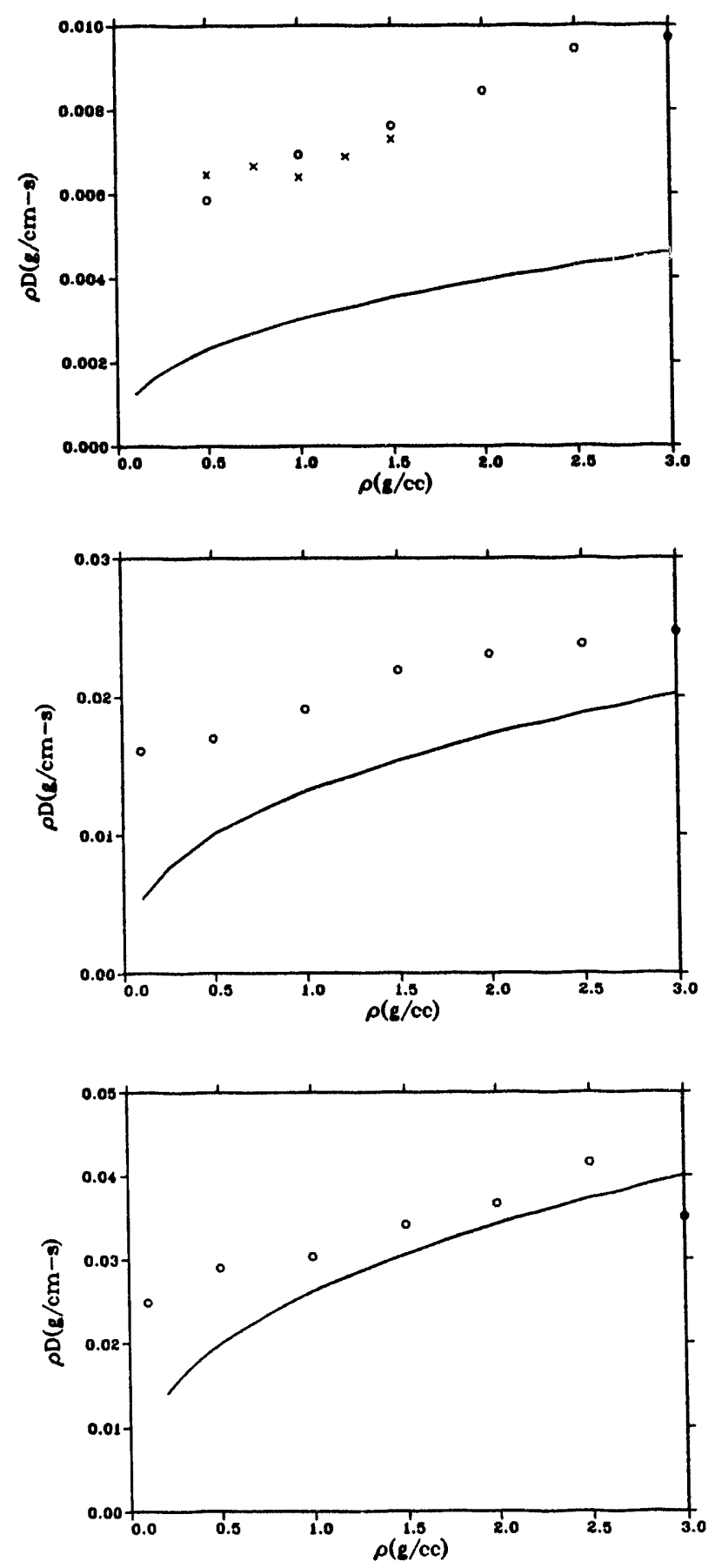

Figure 2: $\rho \mathrm{D}_{H}$ as a function of $\mathrm{T}$ and $\rho: \operatorname{circle}(\operatorname{ss} / \mathrm{TB}), \operatorname{cross}(\mathrm{LDA})$, line(OCP); top: $\mathrm{T}=1 \mathrm{eV}$, middle: $\mathrm{T}=3 \mathrm{eV}$, bottom: $\mathrm{T}=5 \mathrm{eV}$. 


\section{References}

[1] Radiative Properties of Hot Dense Matter, eds. J. Davis, C.Hooper, R. Lee, A. Merts, and B. Rozsnyai, World Scientific, Singapore (1985); Radiative Properties of Hot Dense Matter, eds. B. Roznygai, C. Hooper, R. Cauble, R.Lee, and J. Davis, World Scientific, Singapore (1987); Radiative Properties of Hot Dense Matter, eds. W. Goldstein, C. Hooper, J. Gauthier, J. Seely, and R. Lee, World Scientific, Singapore (1991). J.C. Weishert in Applied Atomic Collision Physics, eds. C.F. Barnett and M.F.A. Harrison, Academic Press, New York (1984).

[2] S. Ichimaru, H. Iyetomi, and S. Tanaka, Phys. Rep. 149, 91 (1987); S. Ichimaru, Rev. Mod. Phys., 541017 (1982); J-P. Hansen, I.R. McDonald, and E.L. Pollock, Phys. Rev. A 11, 1025 (1975).

[3] L.A. Collins and A.L. Merts in Radiative Properties of Hot Dense Matter, p385, eds. B. Roznyai, C. Hooper, R. Bauble, R. Lee, and J. Davis, World Scientific, Singapore (1985).

[4] K. Fujima, T. Watanabe, and H. Adachi, Phys. Rev. A 32,3583 (1985); D.A. Liberman, Phys. Rev. B., $\underline{20}, 4981$ (1979).

[5] S.M. Younger, A.K. Harrison, and G. Sujigama, Phys. Rev. A $\underline{40}, 5256$ (1989); S.M. Younger, Phys. Rev. A., 45, 8657 (1992); S.M. Younger, Radiative Properties of Hot Dense Matter, p137, eds. W. Goldstein, C. Hooper, J. Gauthier, J. Seely and R. Lee, World Scientific, Singapore (1991).

[6] J. Theilhaber, Phys. Fluids B $\underline{4}, 2044$ (1992); Phys. Rev. B 46, 12990 (1992).

[7] I. Kwon, L.A. Collins, J.D. Kress, N. Troullier, and D.L. Lynch, Phys. Rev. Letts.(in press); L.A. Collins, J.D. Kress, D.L. Lynch, and N. Troullier, J. Quant. Spec. Rad. Trans. (Jan. 1994).

[8] D. Hohl, V. Natoli, D.M. Ceperley, and R..M. Martin, Phys. Rev. Lett. $\underline{71} 541$ (1993).

[9] O.F. Sankey and D.J. Niklewski, Phys. Rev. B. 40, 3979 (1989); O.F. Sankey, D.J. Niklewski, D.A. Drabold, and J.D. Dow, Phys. Rev. 14 12750 (1990); J. Broughton and F. Khan, Phys. Rev. B 4 40, 12098 (1989); E. Kaxiras, J. Broughton, and R.J. Hemley, Phys. Rev. Lett. 67, 1138 (1991); C.Z. Wang, C.T. Chan, and K.M. Ho, Phys. Rev. B 39, 8586 (1989).

[10] J. Clerouin, G. Zerah, D. Demisti, and J.-P Hansen, Europhys. Lett, 13, 685 (1990); G. Zerah, J. Clerouin, and E.L. Pollack, Phys. Rev. Lett. 69, 446 (1992).

[11] R. Car and M. Parrinello, Phys. Rev. Lett. 55, 2471 (1985); M.C. Payne, P.D. Bristowe, and J.D. Joannopoulos, Molec. Simul. $\underline{4}, 79$ (1989).

[12] D.L. Lynch, N. Troullier, J.D. Kress, and L.A. Collins, Phys. Rev. B (1994).

[13] H. Gould and J. 'Tobochnik, Computer Simulation Methods, Addison-Wesley, New York (1988).

[14] M.P. Allen and D.J. Tildesley, Computer Simulation of Liquids, Oxford Science Publications, Oxford (1987).

[15] D.K. Remler and P.A. Madden, Molec. Phys. 70, 921 (1990); M.C. Payne, M.P. Teter, D.C. Allen, T.A Arias, and J.I. Joannopoulos, Rev. Mod. Phys. $\underline{64}, 1045$ (1992).

[16] R.G. Parr and W. Yang, Density-Functional Theory of Atoms and Molecules, Oxford University Press, Oxford (1989); J.L. Martins and M.L. Cohen, Phys. Rev. B 37, (6134 (1988). 
[17] R. Hoffman, J. Chem. Phys. 39, 1397 (1963); J.H. Ammeter, H.-B Bürgi, J.C. Thiebolt, and R. Hoffman, J. Am. Chem. Soc, 100, 3686 (1978); G. Calzaferri, L. Forss, and I. Kamber, J. Phys. Chem. 93, 5366 (1989); A.G. Turner, Methods in Molecular Orbital Theory, Prentice-Hall, Englewood Cliffs (1974).

[18] W. Harrison, Electronic Structure and the Properties of Solids, Freeman, San Francisco (1980); J.S. Slater and G.F. Koster, Phys. Rev. 94, 1498 (1984); N.W. Ashcroft and N.D. Mermin, Ch 10, Solid State Physics, Saunders College, Philadelphia (1976).

[19] N. Troullier and J.L. Martins, Phys. Rev. B 43.1993 (1991); J.L. Martins, N. Troullier, and S.-H. Wei, Phys. Rev. B $\underline{43}, 2213$ (1991).

[20] A. Redondo, Tech Note 13, California Institute of Technology, Pasadena (1983); K. Ganguly and R.M. Stratl, J Chem Phys. 95, 4418 (1991).

[21] A.J. Skinner and D.G. Pettifor, J. Phys: Cond. Matter $\underline{3}, 2029$ (1991).

[22] N.F. Mott and E.A. Davis, Electronic Processes in Non-Crystaline Materials, Clarendon Press, Oxford, 1979, Ch. 2; N.F. Mott, Philos, Maj. 19,30 (1969).

[23] R.M. Wentzcovitch and J.L. Martins, Sol. St. Comm. $\underline{78}, 831$ (1991).

[24] S.J. Larson, C. Roxbergh, and A. Lodding, Phys. Chem. Liqs. $\underline{3}, 137$ (1972).

[25] G.-X. Qian, M. Wienert, G.W. Fernando, and J.W. Davenport, Phys. Rev. Lett. $\underline{64}, 1146(1990)$. 

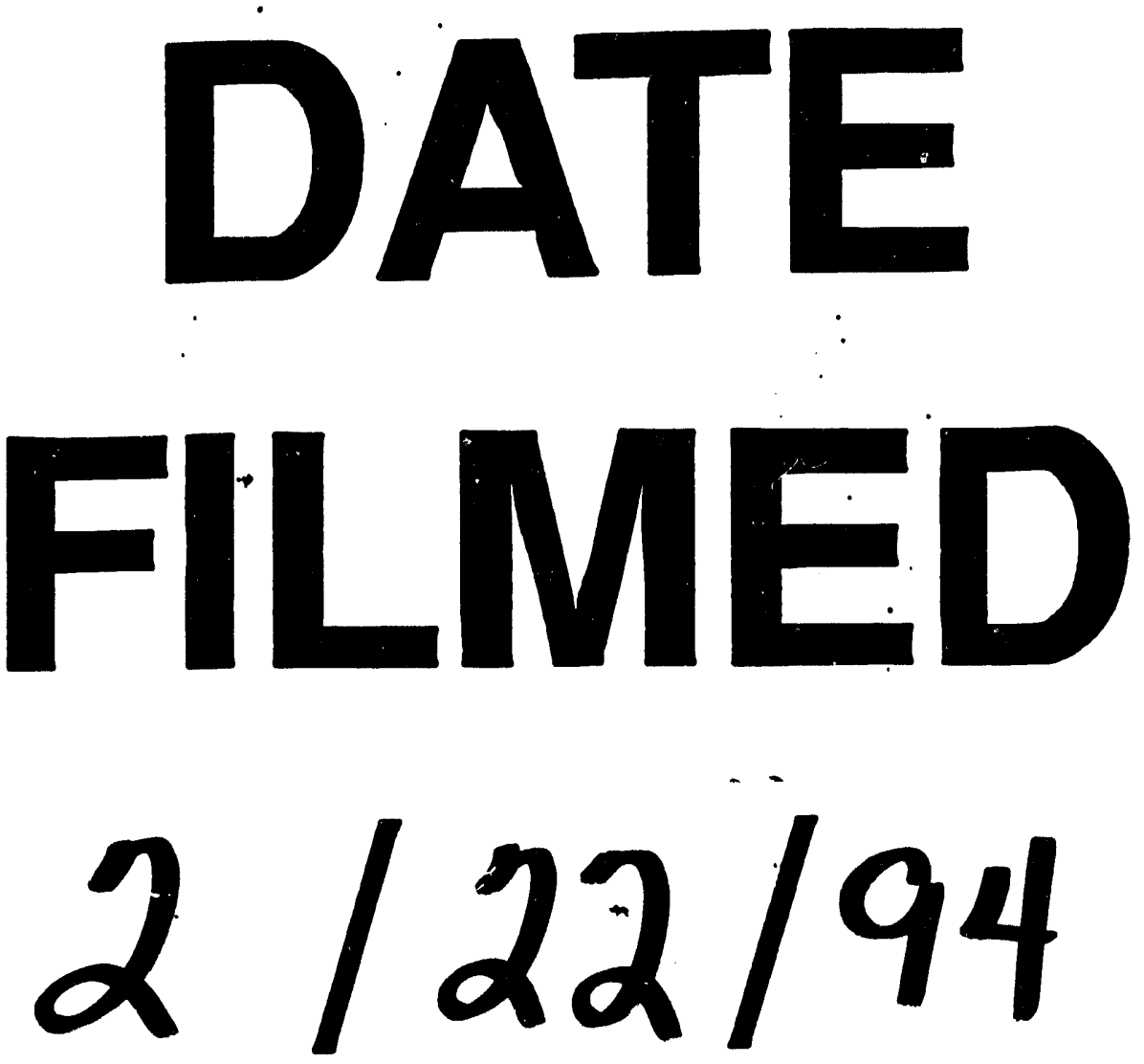

94
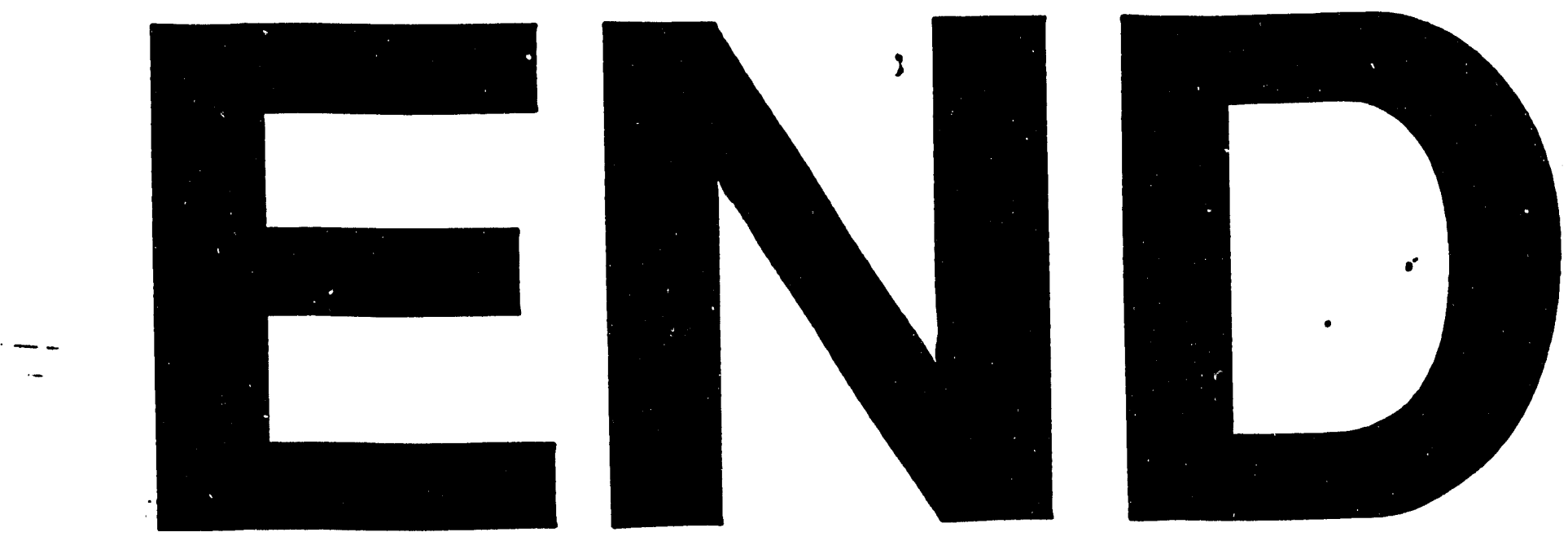


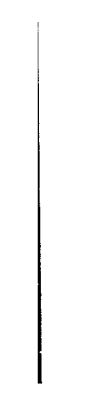

